\title{
Towards a counter-example to a conjecture of De Giorgi in high dimensions
}

Received: January 15, 2002; in final form: June 12, 2003

Published online: April 27, 2004 - (c) Springer-Verlag 2004

\section{Introduction}

In this paper we consider entire solutions to semilinear elliptic equations. We show that solutions that are monotone in one direction are energy minimizers and we discuss existence and uniqueness. Our main result is that the existence of a symmetric global minimizer on $\mathbb{R}^{n-1}$ implies the existence of a counterexample to the conjecture of De Giorgi on $\mathbb{R}^{n}$. This is the analogue in the context of semilinear equations of the result of Bombieri, de Giorgi and Giusti [18] on the existence of non-planar minimal graphs in $\mathbb{R}^{n}$ for $n \geq 9$. Our result is, however, a partial one because it relies on the existence of a symmetric global minimizer, which has not yet been proved. To find such a global minimizer is analogous to the problem in the theory of minimal surfaces of finding the Simons cone.

\subsection{Some analogies between minimal surfaces and a conjecture of De Giorgi}

We recall De Giorgi's conjecture and the analogy between minimal surfaces and solutions of semilinear elliptic equations.

Conjecture 1.1 (De Giorgi [29]). Let us consider a solution $u \in C^{2}\left(\mathbb{R}^{n}\right)$ of

$$
\Delta u=u^{3}-u
$$

D. Jerison: Massachusetts Institute of Technology, Department of Mathematics, Headquarters Office, Building 2, Room 236, 77 Massachusetts Avenue, Cambridge, MA 02139-4307, USA, e-mail: jerison@math.mit.edu

R. Monneau: CERMICS-ENPC, 6-8 avenue B. Pascal, Cité Descartes, Champs-sur-Marne, 77455 Marne-La-Vallée Cedex 2, France, e-mail: monneau@cermics . enpc . fr

* The first author was supported in part by NSF grant DMS 0070412. Part of this work was carried out when the second author was visiting the Department of Mathematics of the Massachusetts Institute of Technology and was supported by a NATO grant. 
such that

$$
|u| \leq 1, \partial_{n} u>0
$$

in the whole $\mathbb{R}^{n}$. Is it true that, for every $\lambda \in \mathbb{R}$, the sets $\{u=\lambda\}$ are hyperplanes, at least if $n \leq 8$ ?

\begin{tabular}{|c|c|c|}
\hline $\mathrm{N}$ ? & $\begin{array}{l}\text { Minimal Surfaces } \\
\text { (Results) }\end{array}$ & $\begin{array}{l}\text { Semilinear Elliptic PDE } \\
\text { (Conjectures) }\end{array}$ \\
\hline 1 & $\begin{array}{l}\text { uniqueness of graph solutions } \\
\qquad \text { (with graph } \subset \mathbb{R}^{n} \text { ) }\end{array}$ & $\begin{array}{l}\text { uniqueness of } x_{n} \text { - monotonic solutions } \\
\text { (defined on cylinders } \omega \times(a, b) \subset \mathbb{R}^{n} \text { ) }\end{array}$ \\
\hline 2 & $\begin{array}{l}\text { by definition } \\
\text { minimize the energy }\end{array}$ & $\begin{array}{l}\text { entire monotonic solutions } \\
\text { are global minimizers }\end{array}$ \\
\hline 3 & $\begin{array}{l}\text { minimal surfaces in } \mathbb{R}^{n} \\
\text { are hyperplanes if } n \leq 7\end{array}$ & $\begin{array}{l}\text { global minimizers on } \mathbb{R}^{n} \\
\text { are planar if } n \leq 7\end{array}$ \\
\hline 4 & $\begin{array}{l}\text { non-hyperplane minimal sets } \\
\text { exist in } \mathbb{R}^{n} \text { for } n \geq 8 \\
\text { (Simons cone) }\end{array}$ & $\begin{array}{l}\text { non-planar global minimizers } \\
\quad \text { exist on } \mathbb{R}^{n} \text { for } n \geq 8\end{array}$ \\
\hline 5 & $\begin{array}{l}\text { minimal graphs in } \mathbb{R}^{n} \\
\text { are hyperplanes if } n \leq 8\end{array}$ & $\begin{array}{c}\text { monotonic solutions on } \mathbb{R}^{n} \\
\text { are planar if } n \leq 8 \\
\text { (De Giorgi conjecture) }\end{array}$ \\
\hline 6 & $\begin{array}{l}\text { non-hyperplane minimal graphs } \\
\text { exist in } \mathbb{R}^{n} \text { for } n \geq 9\end{array}$ & $\begin{array}{l}\text { non-planar monotonic solutions } \\
\text { exists on } \mathbb{R}^{n} \text { for } n \geq 9\end{array}$ \\
\hline 7 & $\begin{array}{l}\text { Lipschitz graphs in } \mathbb{R}^{n} \\
\text { are hyperplanes in all dimensions }\end{array}$ & $\begin{array}{l}\text { monotonic solutions on } \mathbb{R}^{n} \\
\text { with Lipschitz 0-level set } \\
\text { are planar in all dimensions } \\
\text { (Barlow Bass and Gui result) }\end{array}$ \\
\hline 8 & $\begin{array}{l}\text { flat enough minimal surface } \\
\text { in } B_{R} \text { is a graph in } B_{\frac{R}{2}} \\
\text { independently on } R\end{array}$ & $\begin{array}{l}\text { with flat enough } 0 \text {-level set } \\
\text { in } B_{R} \text {, every solution } \\
\text { is a graph in } B_{\frac{R}{2}} \\
\text { independently on } R\end{array}$ \\
\hline
\end{tabular}

In the table, we present some analogies between some well-known facts on minimal surfaces (see [40]) and natural conjectures on solutions to semilinear elliptic partial differential equations.

The conjecture of De Giorgi $C 5$ was proved by Ghoussoub and Gui [37] in dimension $n=2$ and by Ambrosio and Cabré [4] in dimension $n=3$. This conjecture is still open in dimensions $n \geq 4$.

Conjectures $C 1, C 7$ are true: $C 1$ is true by the sliding method, $C 7$ was proved by Barlow, Bass and Gui [10].

Conjecture $C 2$ was first proved under some assumptions by Alberti, Ambrosio and Cabré [2] (see Theorem 1.5). Conjecture $C 2$ is also true under the assump- 
tions of Theorem 1.3 which is proved in the present document. On the contrary, conjecture $C 2$ is not true without any additional assumptions.

Conjecture $C 4$ is still unknown. Nevertheless we prove in Theorem 1.6 that under some simple assumptions $C 4 \Longrightarrow C 6$. For minimal surfaces, conjecture $C 6$ and $C 4$ correspond to the well known results proved by Bombieri, De Giorgi and Giusti [18].

\subsection{On the minimality of monotonic solutions}

In this document we consider bounded solutions $u$ to the following equation:

$$
\Delta u=F^{\prime}(u) \text { on } \mathbb{R}^{n},
$$

where $F \in C^{1,1}(\mathbb{R})$. In particular every such solution is a critical point of the energy

$$
E_{\Omega}(u)=\int_{\Omega} \frac{1}{2}|\nabla u|^{2}+F(u),
$$

where $\Omega$ can be any bounded domain in $\mathbb{R}^{n}$. In order to extend the definition of minimizers of this energy to unbounded domains, we introduce the following definition:

Definition 1.2 (Global minimizers on unbounded domains). Let $\Omega_{0} \subset \mathbb{R}^{n}$ be a domain (not necessary bounded). We say that a function u is a global minimizer on $\Omega_{0}$ iffor every smooth bounded domain $\Omega \subset \subset \Omega_{0}$ we have

$$
\int_{\Omega} \frac{1}{2}|\nabla u|^{2}+F(u) \leq \int_{\Omega} \frac{1}{2}|\nabla v|^{2}+F(v),
$$

for every function $v \in C^{0,1}(\bar{\Omega})$ such that

$$
v=u \text { on } \partial \Omega \text {. }
$$

Then we have:

Theorem 1.3 (Minimality of monotonic solutions). Let there be a function $F \in$ $C^{1,1}(\mathbb{R})$ and $u$ a bounded solution of

$$
\Delta u=F^{\prime}(u) \text { in } \mathbb{R}^{n}
$$

satisfying

$$
\partial_{n} u>0 \text { in } \mathbb{R}^{n} .
$$

Let

$$
\left\{\begin{array}{l}
\bar{u}\left(x^{\prime}\right)=\lim _{x_{n} \rightarrow+\infty} u\left(x^{\prime}, x_{n}\right) \\
\underline{u}\left(x^{\prime}\right)=\lim _{x_{n} \rightarrow-\infty} u\left(x^{\prime}, x_{n}\right) .
\end{array}\right.
$$

Then $u$ is a global minimizer on $\mathbb{R}^{n}$ if and only if $\bar{u}$ and $\underline{u}$ are global minimizers on $\mathbb{R}^{n-1}$.

Proposition 1.4 (Global minimizers are unique local minimizers). Let us assume that $u$ is a global minimizer on $\mathbb{R}^{n}$. Then $u$ is locally the unique minimizer of 
the energy under its own boundary conditions, this means that for every bounded domain $\Omega \subset \mathbb{R}^{n}$ and every function $v \in C^{0,1}(\bar{\Omega})$ we have

$$
\left(\begin{array}{c}
v=u \text { on } \partial \Omega \\
\int_{\Omega} \frac{1}{2}|\nabla v|^{2}+F(v)=\int_{\Omega} \frac{1}{2}|\nabla u|^{2}+F(u)
\end{array}\right) \Longrightarrow(v=u \text { on } \Omega) .
$$

Let us recall the following result:

Theorem 1.5 (Alberti, Ambrosio, Cabré [2]). Let u be a bounded entire solution of (1.1) satisfying (1.2), and let $\Omega \subset \mathbb{R}^{n}$ be a smooth bounded domain. Then

$$
\int_{\Omega} \frac{1}{2}|\nabla u|^{2}+F(u) \leq \int_{\Omega} \frac{1}{2}|\nabla v|^{2}+F(v),
$$

for every function $v \in C^{0,1}(\bar{\Omega})$ such that $v=u$ on $\partial \Omega$ and

$$
\underline{u}\left(x^{\prime}\right) \leq v\left(x^{\prime}, x_{n}\right) \leq \bar{u}\left(x^{\prime}\right) \text { for all } x=\left(x^{\prime}, x_{n}\right) \in \Omega
$$

Theorem 1.5 was proved using results on calibrations for scalar functionals of the calculus of variations. We also give here a different proof of this result based on well-known maximum principle results.

\subsection{Existence of a symmetric global minimizer in $\mathbb{R}^{n-1}$ implies the existence of} a counter-example to the conjecture of De Giorgi in $\mathbb{R}^{n}$

We are now interested in a way of building a "counter-example" to the conjecture of De Giorgi in dimension $n \geq 9$. In this direction we have:

Theorem 1.6 (A strategy to build a counter-example). For the particular nonlinearity $F(v)=\frac{1}{4}\left(v^{2}-1\right)^{2}$, let us assume the existence of a global minimizer $v$ in $\mathbb{R}^{n-1}$ (in the sense of Definition 1.2) which satisfies $|v|<1$ and the following symmetries:

$$
v\left(x_{1}, \ldots,-x_{i}, \ldots, x_{n-1}\right)=v\left(x_{1}, \ldots, x_{i}, \ldots, x_{n-1}\right) \text { for } i=1, \ldots, n-1 .
$$

Then there exists a function $u \in C^{2}\left(\mathbb{R}^{n}\right)$ solution of

$$
\Delta u=u^{3}-u \quad \text { on } \quad \mathbb{R}^{n}
$$

with

$$
|u| \leq 1 \quad \text { and } \quad \partial_{n} u>0
$$

in the whole $\mathbb{R}^{9}$, such that for one $\lambda \in \mathbb{R}$, the set $\{u=\lambda\}$ is not an hyperplane. 
We do not know if this solution satisfies $\lim _{x_{n} \rightarrow+\infty} u\left(x^{\prime}, x_{n}\right)=1$ and $\lim _{x_{n} \rightarrow-\infty} u\left(x^{\prime}, x_{n}\right)=-1$. Neither do we know if such a symmetric global minimizer $v$ as assumed in Theorem 1.6 exists. We expect that we can find such a minimizer in dimension $n=8$, depending only on $|X|,|Y|$ with $X=\left(x_{1}, \ldots, x_{4}\right)$, $Y=\left(x_{5}, \ldots, x_{8}\right)$.

We can now state a generalization of Theorem 1.6 for more general nonlinearities $F$.

Theorem 1.7. Let us consider a function $F \in C^{1,1}(\mathbb{R})$ such that

$$
\begin{cases}F \geq 0 & \text { on } \quad \mathbb{R} \\ F>0 & \text { on } \quad(-1,1) \quad \text { and } \quad F(-1)=F(1)=0 .\end{cases}
$$

Let us assume the existence of a global minimizer $v$ on $\mathbb{R}^{n-1}$ (in the sense of Definition 1.2) which satisfies $|v|<1$ and the following symmetries:

$$
v\left(x_{1}, \ldots,-x_{i}, \ldots, x_{n-1}\right)=v\left(x_{1}, \ldots, x_{i}, \ldots, x_{n-1}\right) \text { for } i=1, \ldots, n-1 .
$$

Then for each $\gamma \in(0, \sqrt{2 F(v(0))})$ there exists a function $u \in C^{2}\left(\mathbb{R}^{n}\right)$ solution to

$$
\Delta u=F^{\prime}(u) \quad \text { on } \quad \mathbb{R}^{n}
$$

such that

$$
|u| \leq 1 \quad \text { and } \quad \partial_{n} u>0
$$

in the whole $\mathbb{R}^{n}$, and for one $\lambda \in \mathbb{R}$, the set $\{u=\lambda\}$ is not an hyperplane.

Moreover, this solution $u$ is a global minimizer on $\mathbb{R}^{n}$, and satisfies

$$
\partial_{n} u(0)=\gamma \quad \text { and } \quad u(0)=v(0)
$$

and the symmetries:

$$
\begin{array}{r}
u\left(x_{1}, \ldots,-x_{i}, \ldots, x_{n-1}, x_{n}\right)=u\left(x_{1}, \ldots, x_{i}, \ldots, x_{n-1}, x_{n}\right) \\
\text { for } i=1, \ldots, n-1 .
\end{array}
$$

Remark 1.8. Looking at the proof of Theorem 1.7, we can see that it gives the existence of a compact connected set of global minimizers on $\mathbb{R}^{n}$ which connects $u_{0}\left(x_{n}\right)$ to $v\left(x^{\prime}\right)$ on $\mathbb{R}^{n}$.

Remark 1.9. The same result holds if we change the symmetries (1.4) by other symmetries which imply $\nabla v(0)=0$.

Remark 1.10. Looking at the proof, we can consider only functions $F \in$ $C^{1,1}([-1,1])$ such that

$$
F>0 \quad \text { on } \quad(-1,1) \quad \text { and } \quad F(-1)=F(1)=F^{\prime}(-1)=F^{\prime}(1)=0 .
$$


Then local perturbations of global minimizers have to be restricted to functions $w$ which satisfy $|w| \leq 1$.

Using the proofs of Theorems 1.6-1.7 we can more generally state the following result:

Proposition 1.11. If $v_{1} \leq v_{2}$ are two global minimizers on $\mathbb{R}^{n}$, then either:

i) there exists a global minimizer $u$ solution to

$$
\Delta u=F^{\prime}(u) \text { on } \mathbb{R}^{n}
$$

with

$$
\partial_{n} u>0
$$

and

$$
v_{1}<u<v_{2}
$$

or,

ii) for each $\lambda \in\left(v_{1}(0), v_{2}(0)\right)$, there exists a global minimizer $v_{\lambda}$ on $\mathbb{R}^{n-1}$ such that $v_{\lambda}(0)=\lambda$ and

$$
v_{1} \leq v_{\lambda}<v_{\lambda^{\prime}} \leq v_{2} \text { if } \lambda<\lambda^{\prime}
$$

\section{Proof of Theorem $\mathbf{1 . 5}$}

Let us consider a bounded solution $u$ of

$$
\Delta u=F^{\prime}(u) \text { on } \mathbb{R}^{n}
$$

satisfying

$$
\partial_{n} u>0 \text { on } \mathbb{R}^{n} \text {. }
$$

Assume that $F \in C^{2}(\mathbb{R})$. Then

$$
\left\{\begin{array}{l}
\bar{u}\left(x^{\prime}\right)=\lim _{x_{n} \rightarrow+\infty} u\left(x^{\prime}, x_{n}\right) \\
\underline{u}\left(x^{\prime}\right)=\lim _{x_{n} \rightarrow-\infty} u\left(x^{\prime}, x_{n}\right)
\end{array}\right.
$$

are bounded solutions of (2.1) on $\mathbb{R}^{n-1}$ such that

$$
\underline{u}<\bar{u} .
$$

Then we have the following result (whose corollary is Theorem 1.5)

Proposition 2.1. If the function $u$ satisfies the above assumptions, then $u$ is locally the unique solution of equation (2.1) in the set of functions $v$ satisfying $\underline{u} \leq v \leq \bar{u}$. This means that for every smooth bounded domain $\Omega \subset \mathbb{R}^{n}$, if we consider a solu- 
tion $v \in C^{0,1}(\bar{\Omega})$ of

$$
\begin{cases}\Delta v=F^{\prime}(v) & \text { on } \Omega \\ v=u & \text { on } \partial \Omega\end{cases}
$$

such that

$$
\underline{u} \leq v \leq \bar{u}
$$

then

$$
v=u \text { on } \Omega \text {. }
$$

We first need the following two lemmata. In particular in the first lemma we recall (without proofs) some well-known results in the spirit of the maximum principle [15]. The second lemma will be proved later.

Lemma 2.2 (Maximum Principle Tools). Let a domain $\Omega \subset \mathbb{R}^{n}$, and a function $w \in C^{0}(\bar{\Omega})$ satisfying

$$
\begin{cases}\Delta w-c(x) w \leq 0 & \text { on } \Omega \\ w \geq 0 & \text { on } \Omega,\end{cases}
$$

where $c$ is a bounded function. Then we have:

\section{i) Strong Maximum Principle:}

$$
\text { Either } w>0 \text { or } w \equiv 0 \text { on } \Omega \text {. }
$$

\section{ii) Harnack Inequality:}

Let a ball $B_{R} \subset \Omega$. Then there exists a constant $C=C\left(|c|_{L^{\infty}(\Omega)}, R\right)$ such that

$$
\sup _{B_{\frac{R}{2}}} w \leq C \inf _{\frac{R}{2}} w .
$$

\section{iii) Hopf Lemma:}

Let $B_{r}\left(X_{1}\right) \subset \Omega$ with $X_{0} \in \partial B_{r}\left(X_{1}\right) \cap \partial \Omega$ and

$$
w\left(X_{0}\right)=0 .
$$

Then

$$
\text { either } \quad w \equiv 0 \quad \text { or } \quad \liminf _{\rho \rightarrow 0^{+}} \frac{1}{\rho} w\left(X_{0}+\rho \frac{X_{1}-X_{0}}{\left|X_{1}-X_{0}\right|}\right)>0 .
$$

iv) Maximum Principle in "narrow domains":

Let us assume that diam $\Omega \leq d_{0}$. There exists $\delta>0$ depending only on $n, d_{0}$ and $|c|_{L^{\infty}(\Omega)}$ such that if meas $\Omega=|\Omega|<\delta$, then for every $v \in C^{0}(\bar{\Omega})$,

$$
\left(\begin{array}{c}
\Delta v-c(x) v \leq 0 \text { on } \Omega \\
v \geq 0 \text { on } \partial \Omega
\end{array}\right) \Longrightarrow v \geq 0 \text { on } \Omega .
$$


Lemma 2.3. Let a ball $B_{R}^{\prime} \subset \mathbb{R}^{n-1}$. Then

$$
\begin{aligned}
& \lim _{x_{n} \rightarrow+\infty} \sup _{x^{\prime} \in B_{R}^{\prime}}\left(\bar{u}\left(x^{\prime}\right)-u\left(x^{\prime}, x_{n}\right)\right)=0 \\
& \lim _{x_{n} \rightarrow-\infty} \sup _{x^{\prime} \in B_{R}^{\prime}}\left(u\left(x^{\prime}, x_{n}\right)-\underline{u}\left(x^{\prime}\right)\right)=0
\end{aligned}
$$

Proof of Lemma 2.3. Let us prove (2.5). The function $w(x)=\bar{u}\left(x^{\prime}\right)-u\left(x^{\prime}, x_{n}\right)$ satisfies

$$
\begin{cases}\Delta w=c(x) w & \text { on } \mathbb{R}^{n} \\ w \geq 0 & \text { on } \mathbb{R}^{n},\end{cases}
$$

where $c(x)$ is bounded as in the proof of Proposition 2.1, Step 1. From Harnack's inequality (Lemma 2.2 ii)) we get by a simple covering argument that, for $B_{R}^{\prime} \subset \mathbb{R}^{n-1}$,

$$
\sup _{B_{R}^{\prime} \times\left\{x_{n}\right\}} w \leq C_{R} \inf _{B_{R}^{\prime} \times\left[x_{n}-R, x_{n}+R\right]} w .
$$

Now we know that for each $x_{0}^{\prime} \in \mathbb{R}^{n-1}, \lim _{x_{n} \rightarrow+\infty} w\left(x_{0}^{\prime}, x_{n}\right)=0$. Choosing one point $x_{0}^{\prime} \in B_{R}^{\prime}$, we deduce (2.5)

$$
\lim _{x_{n} \rightarrow+\infty} \sup _{x^{\prime} \in B_{R}^{\prime}}\left(\bar{u}\left(x^{\prime}\right)-u\left(x^{\prime}, x_{n}\right)\right)=0 .
$$

The proof of (2.6) is similar. This proves the lemma.

Proof of Proposition 2.1. Let us assume that there exists a smooth bounded domain $\Omega \subset \mathbb{R}^{n}$ and a function $v \in C^{0,1}(\bar{\Omega})$ such that

$$
\begin{cases}\Delta v=F^{\prime}(v) & \text { on } \Omega \\ v=u & \text { on } \partial \Omega\end{cases}
$$

with

$$
\underline{u} \leq v \leq \bar{u} \text { on } \Omega
$$

We will prove in three steps that

$$
v \equiv u \text { on } \Omega
$$

Step 1. Construction of a global subsolution.

By the sub- and supersolution method, let us build a function $\bar{v}$ as the minimal supersolution above $v$ and $u$ on $\Omega$. In particular we get, by construction,

$$
\begin{cases}\Delta \bar{v}=F^{\prime}(\bar{v}) & \text { on } \Omega \\ \max (u, v) \leq \bar{v} \leq \bar{u} & \text { on } \Omega \\ \bar{v}=u & \text { on } \partial \Omega\end{cases}
$$


Let us assume that, for some interior point $X_{0} \in \Omega$,

$$
v\left(X_{0}\right)>u\left(X_{0}\right) .
$$

Then let us define

$$
w=\bar{v}-u
$$

which satisfies

$$
\begin{cases}\Delta w=c(x) w & \text { on } \Omega \\ w \geq 0 & \text { on } \Omega \\ w\left(X_{0}\right)>0, & \end{cases}
$$

where

$$
c(x)= \begin{cases}\frac{F^{\prime}\left(\bar{v}\left(x^{\prime}\right)\right)-F^{\prime}(u(x))}{\bar{v}\left(x^{\prime}\right)-u(x)} & \text { if } \bar{v}\left(x^{\prime}\right) \neq u(x) \\ 0 & \text { if } \bar{v}\left(x^{\prime}\right)=u(x) .\end{cases}
$$

Because $F \in C^{2}(\mathbb{R})$ and $\bar{v}, u$ are bounded, the function $c$ is then bounded and the strong maximum principle (Lemma 2.2 i)) implies that $w>0$ on $\Omega$, i.e.

$$
\bar{v}>u \text { on } \Omega \text {. }
$$

Similarly we can prove that

$$
\bar{u}>\bar{v} \text { on } \Omega \text {. }
$$

Moreover the Hopf lemma (Lemma 2.2 iii)) implies that

$$
\frac{\partial}{\partial n}(\bar{v}-u)<0 \text { on } \partial \Omega
$$

and then the function

$$
\tilde{v}(x)= \begin{cases}\bar{v} & \text { on } \Omega \\ u & \text { on } \mathbb{R}^{n} \backslash \Omega\end{cases}
$$

is a subsolution on $\mathbb{R}^{n}$, i.e. satisfies

$$
\Delta \tilde{v} \geq F^{\prime}(\tilde{v}) \text { on } \mathbb{R}^{n}
$$

Step 2. Sliding method.

Let us consider the function

$$
u^{t}(x)=u\left(x^{\prime}, x_{n}+t\right)
$$

By Lemma 2.3 we know that $u\left(x^{\prime}, x_{n}\right) \rightarrow \bar{u}\left(x^{\prime}, x_{n}\right)$ as $x_{n} \rightarrow+\infty$ uniformly for $x^{\prime}$ in a compact set of $\mathbb{R}^{n-1}$. On the other hand, by construction of $\tilde{v}$, we have

$$
\bar{u}>\tilde{v} \text { on } \Omega \text {. }
$$


Because $\Omega$ is bounded we deduce that, for some $t_{0}>0$,

$$
u^{t}>\tilde{v} \text { on } \Omega \text { for } t \geq t_{0} .
$$

Because $\partial_{n} u>0$ we get

$$
u^{t}>\tilde{v} \text { on } \mathbb{R}^{n} \backslash \Omega \text { for } t \geq t_{0} .
$$

Let

$$
t^{*}=\inf \left\{t \geq 0, \forall s \geq t, u^{s} \geq \tilde{v} \text { on } \mathbb{R}^{n}\right\}
$$

then

$$
u^{t^{*}} \geq \tilde{v} \text { on } \mathbb{R}^{n}
$$

If $t^{*}>0$, then

$$
u^{t^{*}}>\tilde{v} \text { on } \mathbb{R}^{n} \backslash \Omega
$$

and we deduce the existence of an interior point $X_{0} \in \Omega$ such that

$$
u^{t^{*}}\left(X_{0}\right)=\tilde{v}\left(X_{0}\right) \text {. }
$$

Using the strong maximum principle (Lemma 2.2 i)) as in Step 1 we conclude that

$$
u^{t^{*}} \equiv \tilde{v} \text { on } \Omega
$$

and then $u^{t^{*}}=u$ on $\partial \Omega$. This is a contradiction because $\partial_{n} u>0$.

Therefore $t^{*}=0$ and $\tilde{v}=u$, i.e. $\bar{v}=u$ on $\Omega$, and then

$$
v \leq u \text { on } \Omega \text {. }
$$

Step 3. Similarly we can repeat Step 1 and 2 with a global supersolution. We then conclude that

$$
v \geq u \text { on } \Omega .
$$

Then $u=v$ on $\Omega$ which proves the proposition.

\section{Proof of Theorem 1.3 and Proposition 1.4}

To prove Proposition 1.4, we first need the following result:

Lemma 3.1. Let a bounded domain $\Omega \subset \mathbb{R}^{n}$ such that two functions $u, v \in$ $C^{0,1}(\bar{\Omega})$ are minimizers of the same energy

$$
E_{\Omega}(w):=\int_{\Omega} \frac{1}{2}|\nabla w|^{2}+F(w),
$$

with the same boundary condition

$$
u=v \text { on } \partial \Omega .
$$

Then either $v<u$ or $v>u$ or $v \equiv u$ on $\Omega$. 
Proof of Lemma 3.1.

Step 1. Let us assume that $\Omega_{-}:=\{v<u\} \neq \emptyset$. We claim that

$$
E_{\Omega_{-}}(v)=E_{\Omega_{-}}(u) .
$$

If not, let us assume, for example, that

$$
E_{\Omega_{-}}(v)<E_{\Omega_{-}}(u) .
$$

Now define a new candidate as a minimizer

$$
v_{-}= \begin{cases}v & \text { on } \Omega_{-} \\ u & \text { on } \Omega \backslash \Omega_{-} .\end{cases}
$$

Then we have

$$
E_{\Omega}\left(v_{-}\right) \leq E_{\Omega_{-}}(v)+E_{\Omega \backslash \Omega_{-}}(u)<E_{\Omega}(u),
$$

which gives a contradiction with the minimality of $u$.

Assuming that

$$
E_{\Omega_{-}}(v)>E_{\Omega_{-}}(u),
$$

we get a similar contradiction considering

$$
v_{+}= \begin{cases}u & \text { on } \Omega_{-} \\ v & \text { on } \Omega \backslash \Omega_{-} .\end{cases}
$$

Step 2. We then have

$$
\left\{\begin{array}{l}
E_{\Omega}\left(v_{-}\right)=E_{\Omega}(u) \\
v_{-}=u \text { on } \partial \Omega \\
v_{-} \leq u \text { on } \Omega
\end{array}\right.
$$

We then conclude from the strong maximum principle (Lemma $2.2 \mathrm{i}$ )) that either $v_{-} \equiv u$ on $\Omega$, which is impossible by construction, or $v_{-}<u$ on $\Omega$, which means

$$
v<u \text { on } \Omega \text {. }
$$

Step 3. If we assume that $\Omega_{+}:=\{v>u\} \neq \emptyset$ we prove similarly that

$$
v>u \text { on } \Omega \text {. }
$$

This ends the proof of the lemma.

Proof of Proposition 1.4. Let us assume that $u$ is a global minimizer on $\mathbb{R}^{n}$, but locally not unique, i.e. there exist a bounded domain $\Omega \subset \mathbb{R}^{n}$ and a function $v \in C^{0,1}(\bar{\Omega}), v \not \equiv u$ such that

$$
v=u \text { on } \partial \Omega
$$

and

$$
E_{\Omega}(v)=E_{\Omega}(u) .
$$

We will prove a contradiction in two steps. 
Step 1. By Lemma 3.1, let us assume that $v<u$ on $\Omega$ (the case $v>u$ can be treated similarly). From the Hopf lemma (Lemma 2.2 iii)), we deduce that the function

$$
w=u-v
$$

satisfies

$$
\liminf _{\rho \rightarrow 0^{+}} \frac{1}{\rho} w\left(X_{0}+\rho \frac{X_{1}-X_{0}}{\left|X_{1}-X_{0}\right|}\right)>0,
$$

for $B_{r}\left(X_{1}\right) \subset \Omega$ with $X_{0} \in \partial B_{r}\left(X_{1}\right) \cap \partial \Omega$.

Step 2. Let us consider a ball $B_{R}$ which strictly contains $\Omega$ :

$$
B_{R} \supset \supset \Omega \text {. }
$$

Then the extension $\tilde{v}$ of $v$ to $B_{R}$ defined by

$$
\tilde{v}= \begin{cases}v & \text { on } \Omega \\ u & \text { on } B_{R} \backslash \Omega\end{cases}
$$

satisfies

$$
E_{\Omega}(\tilde{v})=E_{\Omega}(u)
$$

and then is a minimizer on $B_{R}$ and satisfies the Euler-Lagrange equation

$$
\Delta \tilde{v}=F^{\prime}(\tilde{v}) \text { on } B_{R}
$$

By the standard elliptic estimates, we deduce that $\tilde{v} \in C^{2}\left(B_{R}\right)$, which is in contradiction with (3.1) and the fact that $u \in C^{2}\left(B_{R}\right)$.

To prove Theorem 1.3, we first need the following lemma which will be proved later:

Lemma 3.2. Let a domain $\Omega_{0} \subset \mathbb{R}^{n-1}$ (not necessary bounded). Let a function $w$ be defined on $\Omega_{0}$. Then $w$ is a global minimizer on $\Omega_{0} \times \mathbb{R}$ if and only if $w$ is a global minimizer on $\Omega_{0}$.

Proof of Lemma 3.2.

i) $w$ global minimizer on $\Omega_{0} \Longrightarrow w$ global minimizer on $\Omega_{0} \times \mathbb{R}$.

Let us consider a smooth bounded domain $\Omega \subset \Omega_{0} \times \mathbb{R}$, and let us assume that $w$ is not a minimizer of the energy on $\Omega$, i.e. there exists a function $v \in C^{0,1}(\bar{\Omega})$, $v \not \equiv w$ such that

$$
v\left(x^{\prime}, x_{n}\right)=w\left(x^{\prime}\right) \text { on } \partial \Omega
$$

and

$$
E_{\Omega}(v)<E_{\Omega}(w):=\int_{\Omega} \frac{1}{2}|\nabla w|^{2}+F(w) .
$$


Then we consider a cylindrical open set $\Omega_{R}:=\Omega_{R}^{\prime} \times(-R, R)$ which contains $\Omega$ :

$$
\Omega_{R} \supset \supset,
$$

where $\Omega_{R}^{\prime} \subset \subset \Omega_{0}$ is a smooth bounded domain. We define the extension $v_{R}$ of the function $v$ to $\Omega_{R}$ by

$$
v_{R}(x)= \begin{cases}v & \text { on } \Omega \\ w & \text { on } \Omega_{R} \backslash \Omega .\end{cases}
$$

In particular $v_{R}$ satisfies

$$
v_{R}\left(x^{\prime}, x_{n}\right)=w\left(x^{\prime}\right) \text { on } \partial \Omega_{R} .
$$

As a consequence of (3.2) we get

$$
E_{\Omega_{R}}\left(v_{R}\right) \leq E_{\Omega_{R} \backslash \Omega}(w)+E_{\Omega}(v)<E_{\Omega_{R}}(w),
$$

i.e.

$$
\int_{-R}^{R} d x_{n} \int_{\Omega_{R}^{\prime}} \frac{1}{2}\left|\nabla v_{R}\right|^{2}+F\left(v_{R}\right)<\int_{-R}^{R} d x_{n} \int_{\Omega_{R}^{\prime}} \frac{1}{2}|\nabla w|^{2}+F(w) .
$$

This implies that at least one section $v_{R, t}$, for some $t \in(-R, R)$,

$$
v_{R, t}\left(x^{\prime}\right):=v_{R}\left(x^{\prime}, x_{n}=t\right)
$$

satisfies

$$
E_{\Omega_{R}^{\prime}}\left(v_{R, t}\right)<E_{\Omega_{R}^{\prime}}(w)
$$

Moreover

$$
v_{R, t}=w \text { on } \partial \Omega_{R}^{\prime},
$$

which by the minimality of $w$ on $\Omega_{R}^{\prime} \subset \mathbb{R}^{n-1}$ gives a contradiction.

ii) $w$ global minimizer on $\Omega_{0} \times \mathbb{R} \Longrightarrow w$ global minimizer on $\Omega_{0}$.

Let us assume that $w$ is not a global minimizer on $\Omega_{0}$, i.e. there exists a smooth bounded domain $\Omega^{\prime} \subset \subset \Omega_{0}$ and a function $v \in C^{0,1}\left(\overline{\Omega^{\prime}}\right)$ such that

$$
E_{\Omega^{\prime}}(v)<E_{\Omega^{\prime}}(w) \text { and } v=w \text { on } \partial \Omega^{\prime} .
$$

Then let $\psi \in \mathscr{D}(\mathbb{R})$ such that

$$
\left\{\begin{array}{lll}
\psi=1 & \text { on } & (-t, t) \\
\psi=0 & \text { on } & \mathbb{R} \backslash(-t-1, t+1) .
\end{array}\right.
$$

We then define

$$
u\left(x^{\prime}, x_{n}\right)=\psi\left(x_{n}\right) v\left(x^{\prime}\right)+\left(1-\psi\left(x_{n}\right)\right) w\left(x^{\prime}\right)
$$


and let $\Omega \subset \Omega^{\prime} \times \mathbb{R}$ be any smooth bounded domain such that

$$
\Omega \supset \Omega^{\prime} \times(-t-1, t+1) .
$$

Because we can find a constant $C$ such that

$$
\left|E_{\Omega^{\prime} \times((-t-1,-t) \cup(t, t+1))}(w)-E_{\Omega^{\prime} \times((-t-1,-t) \cup(t, t+1))}(u)\right| \leq C,
$$

we get

$$
E_{\Omega}(w)-E_{\Omega}(u) \geq-C+2 t\left(E_{\Omega^{\prime}}(w)-E_{\Omega^{\prime}}(v)\right)>0,
$$

which is strictly positive for $t$ large enough. Because $u=w$ on $\partial \Omega$, we then get a contradiction with the fact that $w$ is a minimizer on $\Omega \subset \Omega_{0} \times \mathbb{R}$.

This ends the proof of the lemma.

Proof of Theorem 1.3.

i) $\bar{u}, \underline{u}$ global minimizers $\Longrightarrow u$ global minimizer.

Let us consider a smooth bounded domain $\Omega \subset \mathbb{R}^{n}$ and a function $v$ which minimizes the energy

$$
E_{\Omega}(v):=\int_{\Omega} \frac{1}{2}|\nabla v|^{2}+F(v)
$$

among all functions in $C^{0,1}(\bar{\Omega})$ with

$$
v=u \text { on } \partial \Omega .
$$

It is easy to see that such a function $v$ exists. We will prove that

$$
v \equiv u \text { on } \Omega \text {. }
$$

To this end let us introduce

$$
\begin{aligned}
& \Omega_{+}=\{x \in \Omega, v>\bar{u}\} \\
& \Omega_{-}=\{x \in \Omega, v<\underline{u}\} .
\end{aligned}
$$

Because we have

$$
\underline{u}<u<\bar{u}
$$

we get

$$
\begin{aligned}
& \Omega_{+} \subset \subset \Omega \\
& \Omega_{-} \subset \subset \Omega
\end{aligned}
$$

and a natural candidate to minimize the energy is then

$$
\tilde{v}= \begin{cases}\underline{u} & \text { on } \Omega_{-} \\ v & \text { on } \Omega \backslash\left(\Omega_{-} \cup \Omega_{+}\right) \\ \bar{u} & \text { on } \Omega_{+} .\end{cases}
$$


Applying Lemma 3.2 to the function $\bar{u}$ (resp. $\underline{u}$ ), we get that $\bar{u}$ (resp. $\underline{u}$ ) is a global minimizer on $\mathbb{R}^{n}$. From Proposition 1.4 , we deduce that $\bar{u}$ (resp. $\underline{u}$ ) is the unique minimizer of the energy under its own boundary conditions on $\Omega_{+}$(resp. on $\Omega_{-}$), and then

$$
E_{\Omega}(v)>E_{\Omega}(\tilde{v}) \text { if } \Omega_{-} \neq \emptyset \text { or } \Omega_{+} \neq \emptyset .
$$

By the minimality of $v$ we conclude that $\Omega_{-}=\Omega_{+}=\emptyset$ and then

$$
\underline{u} \leq v \leq \bar{u} .
$$

We then apply Proposition 2.1 which proves that $v=u$ on $\Omega$.

ii) u global minimizer $\Longrightarrow \bar{u}, \underline{u}$ global minimizers.

By definition of $\bar{u}, \underline{u}$ it is clear that there are global minimizers on $\mathbb{R}^{n}$. Then Lemma 3.2 implies that $\bar{u}, \underline{u}$ are also global minimizers on $\mathbb{R}^{n-1}$.

This ends the proof of the theorem.

\section{Proof of Theorem 1.7}

To prove Theorem 1.7 we will need many lemmata. We start with the following:

Lemma 4.1. Let $u$ and $v$ be two global minimizers on $\Sigma=B_{R}^{\prime} \times \mathbb{R}$ such that

$$
u \geq v \quad \text { on } \quad \partial \Sigma,
$$

satisfying moreover

$$
\lim _{\lambda \rightarrow+\infty} \sup _{\begin{array}{r}
x^{\prime} \in B_{R}^{\prime} \\
\left|x_{n}\right| \geq \lambda
\end{array}}(|u-v|+|\nabla(u-v)|)=0 .
$$

Then either $u<v$ or $u>v$ or $u=v$.

Proof of Lemma 4.1. For $h>0$ let us introduce

$$
\Sigma_{h}:=\Sigma \cap\left\{\left|x_{n}\right|<h\right\},
$$

and let us define

$$
\tilde{u}=\left\{\begin{array}{lll}
u & \text { on } & \{u \geq v\} \\
v & \text { on } & \{u<v\} .
\end{array}\right.
$$

Step 1. Let us consider

$$
\liminf _{h \rightarrow+\infty}\left(E_{\Sigma_{h}}(\tilde{u})-E_{\Sigma_{h}}(u)\right)=\underline{a} \in \overline{\mathbb{R}}
$$

Let us assume that $\underline{a}<0$. Then there exists a constant $C>0$ and a sequence $\left\{h_{k}\right\}_{k \in \mathbb{N}}$ such that $h_{k} \rightarrow+\infty$ and

$$
E_{\Sigma_{h_{k}}}(\tilde{u})-E_{\Sigma_{h_{k}}}(u) \leq-C<0 .
$$


Now let $\psi_{k} \in \mathscr{D}(\mathbb{R})$ such that

$$
\left\{\begin{array}{lll}
\psi=1 & \text { on } & \left(-h_{k}, h_{k}\right) \\
\psi=0 & \text { on } & \mathbb{R} \backslash\left(-h_{k}-1, h_{k}+1\right),
\end{array}\right.
$$

and let

$$
\tilde{u}_{k}(x)=\psi_{k}\left(x_{n}\right) \tilde{u}(x)+\left(1-\psi_{k}\left(x_{n}\right)\right) u(x) .
$$

Then we can find a smooth bounded domain $\Omega_{k} \subset \Sigma$ such that

$$
\Omega_{k} \supset \Sigma_{h_{k}+1} \text {. }
$$

Then

$$
E_{\Omega_{k}}\left(\tilde{u}_{k}\right)-E_{\Omega_{k}}(u)=E_{\Sigma_{h_{k}+1}}\left(\tilde{u}_{k}\right)-E_{\Sigma_{h_{k}+1}}(u) \leq c_{k}-C,
$$

where $c_{k}$ is a constant such that

$$
\left|E_{\Sigma_{h_{k}+1} \backslash \Sigma_{h_{k}}}\left(\tilde{u}_{k}\right)-E_{\Sigma_{h_{k}+1} \backslash \Sigma_{h_{k}}}(u)\right| \leq c_{k} .
$$

But because $|u-v|+|\nabla(u-v)| \rightarrow 0$ as $\left|x_{n}\right|$ tends to $+\infty$, we get

$$
c_{k} \rightarrow 0
$$

and then

$$
E_{\Omega_{k}}\left(\tilde{u}_{k}\right)-E_{\Omega_{k}}(u)<-C / 2,
$$

for $k$ large enough. Because $\tilde{u}_{k}=u$ on $\partial \Omega_{k}$, we get a contradiction with the minimality of $u$. Therefore

$$
\underline{a} \geq 0
$$

Step 2. We now consider

$$
\limsup _{h \rightarrow+\infty}\left(E_{\Sigma_{h}}(\tilde{u})-E_{\Sigma_{h}}(u)\right)=\bar{a} \in \overline{\mathbb{R}} .
$$

We simply remark that

$$
E_{\Sigma_{h}}(\tilde{u})-E_{\Sigma_{h}}(u)=-\left(E_{\Sigma_{h}}(\tilde{v})-E_{\Sigma_{h}}(v)\right),
$$

where

$$
\tilde{v}=\left\{\begin{array}{lll}
v & \text { on } & \{u \geq v\} \\
u & \text { on } & \{u<v\} .
\end{array}\right.
$$

We can then apply Step 1 to $\tilde{v}$ and $v$ and we get

$$
\bar{a} \leq 0 \text {. }
$$

We then have

$$
\lim _{h \rightarrow+\infty}\left(E_{\Sigma_{h}}(\tilde{u})-E_{\Sigma_{h}}(u)\right)=0 .
$$


Step 3. Let $\phi \in \mathscr{D}(\Sigma)$ and $h_{0}>0$ such that

$$
\operatorname{supp}(\phi) \subset \Sigma_{h_{0}} .
$$

Then for every $h \geq h_{0}$ :

$$
\begin{aligned}
E_{\Sigma_{h_{0}}}(\tilde{u}+\phi)-E_{\Sigma_{h_{0}}}(\tilde{u}) & =E_{\Sigma_{h}}(\tilde{u}+\phi)-E_{\Sigma_{h}}(\tilde{u}) \\
& =\left(E_{\Sigma_{h}}(\tilde{u}+\phi)-E_{\Sigma_{h}}(u)\right)+\left(E_{\Sigma_{h}}(\tilde{u})-E_{\Sigma_{h}}(u)\right) .
\end{aligned}
$$

As in Step 1 we deduce that

$$
\liminf _{h \rightarrow+\infty}\left(E_{\Sigma_{h}}(\tilde{u}+\phi)-E_{\Sigma_{h}}(u)\right) \geq 0,
$$

and then by (4.1) we have

$$
E_{\Sigma_{h_{0}}}(\tilde{u}+\phi)-E_{\Sigma_{h_{0}}}(\tilde{u}) \geq 0 .
$$

Now because it is true for every $\phi \in \mathscr{D}(\Sigma)$ with $h_{0}$ satisfying (4.2), we deduce that $\tilde{u}$ has to statisfy the Euler-Lagrange equation

$$
\Delta \tilde{u}=F^{\prime}(\tilde{u}) \quad \text { on } \quad \Sigma .
$$

Because $\tilde{u} \geq u$, we deduce from the strong maximum principle (Lemma 2.2 i)) that either $\tilde{u}>u$ or $\tilde{u}=u$, i.e.

$$
v>u \text { or } \quad v \leq u
$$

Similarly we get that

$$
u>v \text { or } u \leq v
$$

This proves the lemma.

Lemma 4.2. Let us consider global minimizers $u$ on $\Sigma=B_{R}^{\prime} \times \mathbb{R}$ solutions to the following equations:

$$
\begin{cases}\Delta u=F^{\prime}(u) & \text { on } \Sigma \\ u=g & \text { on } \partial \Sigma,\end{cases}
$$

where $g \in C^{1}(\partial \Sigma)$ satisfies

$$
\partial_{n} g>0 \text { on } \partial \Sigma_{R}
$$

If we assume the following limits at infinity:

$$
\left\{\begin{array}{l}
\lim _{x_{n} \rightarrow+\infty} \sup _{x^{\prime} \in B_{R}^{\prime}}\left(\left|1-u\left(x^{\prime}, x_{n}\right)\right|+\left|\nabla u\left(x^{\prime}, x_{n}\right)\right|\right)=0 \\
\lim _{x_{n} \rightarrow-\infty} \sup _{x^{\prime} \in B_{R}^{\prime}}\left(\left|-1-u\left(x^{\prime}, x_{n}\right)\right|+\left|\nabla u\left(x^{\prime}, x_{n}\right)\right|\right)=0,
\end{array}\right.
$$

then these solutions $u$ are unique. 
Proof of Lemma 4.2. Let us assume that there exists two global minimizers $u$ and $v$ on $\Sigma=B_{R}^{\prime} \times \mathbb{R}$ with $u=v=g$ on $\partial \Sigma$. For $t \in \mathbb{R}$ let us define

$$
u^{t}\left(x^{\prime}, x_{n}\right)=u\left(x^{\prime}, x_{n}+t\right) .
$$

Because $\partial_{n} g>0$ on $\partial \Sigma$, for $t>0$, we have

$$
u^{t}>v \text { on } \partial \Sigma \text {. }
$$

From Lemma 4.1 we deduce that

$$
u^{t}>v \text { on } \Sigma
$$

and then in the limit $t \rightarrow 0$

$$
u \geq v \quad \text { on } \quad \Sigma \text {. }
$$

Similarly we get

$$
v \geq u \quad \text { on } \quad \Sigma,
$$

which proves the uniqueness of the solution $u=v$. This ends the proof of the lemma.

Let us recall that, in Theorem 1.7, we assume the existence of a function $v\left(x^{\prime}\right)$ on $\mathbb{R}^{n-1}$. We will need to connect on $\mathbb{R}^{n}$ the two particular solutions $u_{0}\left(x_{n}\right)$ and $v\left(x^{\prime}\right)$ by a continuous curve of solutions on $\mathbb{R}^{n}$. To this end we introduce the following functions for $t \in[0,1]$ :

$$
U_{t}\left(x^{\prime}, x_{n}\right):=u_{0}\left(t u_{0}^{-1}\left(v\left(x^{\prime}\right)\right)+(1-t) x_{n}\right),
$$

which satisfies, in particular,

$$
\left\{\begin{array}{l}
U_{0}\left(x^{\prime}, x_{n}\right)=u_{0}\left(x_{n}\right) \\
U_{1}\left(x^{\prime}, x_{n}\right)=v\left(x^{\prime}\right) .
\end{array}\right.
$$

Moreover, the functions $U_{t}$ satisfy

$$
\partial_{n} U_{t} \geq 0
$$

and the same symmetries as $v$, namely (1.5). For each $t \in[0,1]$, we first consider possible solutions $u_{t}$ satisfying on $\Sigma=B_{R}^{\prime}(0) \times \mathbb{R}$

$$
\left\{\begin{array}{lll}
\Delta u_{t}=F^{\prime}\left(u_{t}\right) & \text { on } & \Sigma \\
u_{t}=U_{t} & \text { on } & \partial \Sigma \\
\partial_{n} u_{t} \geq 0 & \text { on } & \Sigma \\
\left|u_{t}\right| \leq 1 & \text { on } & \Sigma .
\end{array}\right.
$$

We have:

Proposition 4.3 (Uniqueness). For each $t \in[0,1]$, there is at most one solution $u_{t}$ of (4.3) which is a global minimizer on $\Sigma$. 
Proposition 4.4 (Existence). For each $t \in[0,1]$, there exists at least one solution $u_{t}$ of (4.3) which is a global minimizer on $\Sigma$.

Proof of Proposition 4.3 (Uniqueness). To simplify the notations let us denote $u_{t}$ by $u$. Let us introduce the limits at infinity:

$$
\begin{aligned}
& \bar{u}\left(x^{\prime}\right)=\lim _{x_{n} \rightarrow+\infty} u\left(x^{\prime}, x_{n}\right) \\
& \underline{u}\left(x^{\prime}\right)=\lim _{x_{n} \rightarrow-\infty} u\left(x^{\prime}, x_{n}\right) .
\end{aligned}
$$

From the monotonicity of $u$ we deduce

$$
\underline{u} \leq u \leq \bar{u} \text { on } \Sigma \text {. }
$$

i) In the case $t=1$, we have

$$
u\left(x^{\prime}, x_{n}\right)=v\left(x^{\prime}\right) \text { on } \partial \Sigma .
$$

In particular $\bar{u}$ satisfies

$$
\begin{cases}\Delta \bar{u}=F^{\prime}(\bar{u}) & \text { on } B_{R}^{\prime} \\ \bar{u}=v & \text { on } \partial B_{R}^{\prime} .\end{cases}
$$

From the minimality of $v$ and from Proposition 1.4 we deduce the uniqueness of the solution to $(4.5)$, i.e.

$$
\bar{u} \equiv v
$$

Similarly,

$$
\underline{u} \equiv v
$$

and from (4.4) we have

$$
u\left(x^{\prime}, x_{n}\right)=v\left(x^{\prime}\right) \text { on } \Sigma \text {. }
$$

ii) When $t \in[0,1), \bar{u}$ satifies

$$
\begin{cases}\Delta \bar{u}=F^{\prime}(\bar{u}) & \text { on } B_{R}^{\prime} \\ \bar{u}=1 & \text { on } \partial B_{R}^{\prime}\end{cases}
$$

Using the fact that the constant 1 is a global minimizer on $\mathbb{R}$, we deduce from Lemma 3.2 that the constant 1 is also a global minimizer on $\mathbb{R}^{n-1}$. We then conclude from Proposition 1.4 to the uniqueness of the solution to (4.6), i.e.

$$
\bar{u} \equiv 1
$$

and then

$$
\lim _{x_{n} \rightarrow+\infty} \sup _{x^{\prime} \in B_{R}^{\prime}}\left(\left|1-u\left(x^{\prime}, x_{n}\right)\right|+\left|\nabla u\left(x^{\prime}, x_{n}\right)\right|\right)=0,
$$


where the convergence on the gradient is a consequence of standard elliptic estimates and the smoothness of $U_{t}$ on the boundary $\partial \Sigma$. Similarly

$$
\lim _{x_{n} \rightarrow-\infty} \sup _{x^{\prime} \in B_{R}^{\prime}}\left(\left|-1-u\left(x^{\prime}, x_{n}\right)\right|+\left|\nabla u\left(x^{\prime}, x_{n}\right)\right|\right)=0 .
$$

We now apply Lemma 4.2, which proves the uniqueness of the solution $u$ on $\Sigma$. This ends the proof of the proposition.

Proof of Proposition 4.4 (Existence). We prove the existence in two steps. We first build a solution on a cylinder of finite length and then get a solution on an infinite cylinder taking a limit when the length of the cylinder tends to infinity.

Step 1. Minimization on finite cylinders. For $h>1$, let us consider the bounded cylinder

$$
\Sigma_{h}:=B_{R}^{\prime} \times(-h, h),
$$

where $B_{R}^{\prime}=\left\{x^{\prime},\left|x^{\prime}\right|<R\right\} \subset \mathbb{R}^{n-1}$ with $x^{\prime}=\left(x_{1}, \ldots, x_{n-1}\right)$. We then consider the minimization of the energy

$$
E_{\Sigma_{h}}(w)=\int_{\Sigma_{h}} \frac{1}{2}|\nabla w|^{2}+F(w)
$$

among all functions $w \in H^{1}\left(\Sigma_{h}\right)$ with the boundary conditions

$$
\begin{cases}w=1 & \text { on }\left\{x_{n}=h\right\} \\ w=-1 & \text { on }\left\{x_{n}=-h\right\} \\ w=U_{t, h} & \text { on } \partial B_{R}^{\prime} \times(-h, h)\end{cases}
$$

where the function $U_{t, h}$ is an approximation of

$$
U_{t}\left(x^{\prime}, x_{n}\right):=u_{0}\left(t u_{0}^{-1}\left(v\left(x^{\prime}\right)\right)+(1-t) x_{n}\right) .
$$

The disadvantage of the function $U_{t}$ is that it does not satisfy the boundary conditions stated in the two first lines of (4.7). To avoid this difficulty we introduce the approximations $U_{t, h}$ of $U_{t}$ which satisfies

$$
U_{t, h} \in C^{0,1}\left(\overline{\Sigma_{h}}\right)
$$

and has still the following properties:

$$
\begin{cases}\partial_{n} U_{t, h} \geq 0 & \text { on } \quad \Sigma_{h} \\ U_{t, h}\left(x_{1}, \ldots,-x_{i}, \ldots, x_{n-1}, x_{n}\right)=U_{t, h}\left(x_{1}, \ldots, x_{i}, \ldots, x_{n-1}, x_{n}\right) \\ & \text { for } i=1, \ldots, n-1 \\ U_{t, h}=U_{t} & \text { on } \Sigma_{h} \cap\left\{-h+1 \leq x_{n} \leq h-1\right\} .\end{cases}
$$


We can, for example, define the function $U_{t, h}$ on $\overline{\Sigma_{h}}$ by

$U_{t, h}= \begin{cases}s+(1-s) U_{t}\left(x^{\prime}, x_{n}=h-1\right) & \text { on } B_{R}^{\prime} \times\{h-1+s\} \text { for } s \in[0,1] \\ U_{t} & \text { on } B_{R}^{\prime} \times(-h+1, h-1) \\ -s+(1-s) U_{t}\left(x^{\prime}, x_{n}=-h+1\right) & \text { on } B_{R}^{\prime} \times\{-h+1-s\} \text { for } s \in[0,1] .\end{cases}$

From the regularity property (4.8) of $U_{t, h}$ we deduce in particular that

$$
E_{\Sigma_{h}}\left(U_{t, h}\right)<+\infty
$$

This implies the existence of a minimizer $u_{t, h}$ of the energy, which in particular satisfies the Euler-Lagrange equation

$$
\Delta u_{t, h}=F^{\prime}\left(u_{t, h}\right) \text { on } \Sigma_{h},
$$

with the previous boundary conditions.

Moreover using a sliding method, we will prove (just below) the following result:

Lemma 4.5. Under the previous assumptions, we have

$$
\partial_{n} u_{t, h} \geq 0 \text { on } \Sigma_{h} .
$$

Step 2. The limit on infinite cylinders. The standard elliptic estimates allow us to take the limit $h \rightarrow+\infty$, and give the existence of a solution $u_{t}$ to

$$
\begin{cases}\Delta u_{t}=F^{\prime}\left(u_{t}\right) & \text { on } \Sigma:=B_{R}^{\prime} \times \mathbb{R} \\ u_{t}=U_{t} & \text { on } \partial \Sigma \\ \partial_{n} u_{t} \geq 0 & \text { on } \Sigma\end{cases}
$$

Moreover, this solution $u_{t}$ is a global minimizer on $\Sigma$. This ends the proof of the proposition.

Proof of Lemma 4.5. We will prove this lemma using a sliding method. To simplify the notations let us denote $u_{t, h}$ by $u$ and $\Sigma_{h}$ by $\Sigma$. Let

$$
u^{t}\left(x^{\prime}, x_{n}\right)=u\left(x^{\prime}, x_{n}+t\right) \quad \text { defined on } \quad \Sigma^{t}:=B_{R}^{\prime} \times(-h-t, h-t) .
$$

Let

$$
w^{t}=u^{t}-u \quad \text { on } \quad \Sigma^{t} \cap \Sigma,
$$

which satisfies

$$
\Delta w^{t}-c^{t}(x) w^{t}=0 \quad \text { on } \quad \Sigma^{t} \cap \Sigma
$$

for some bounded function $c^{t}$. 
Step 1. Initialization. We have $\Sigma^{t} \cap \Sigma \subset\left\{-h \leq x_{n} \leq-h+\delta_{0}\right\}$ for $t \in$ $\left(2 h-\delta_{0}, 2 h\right)$. As a consequence for $\delta_{0}$ small enough, the maximum principle in "narrow domains" (Lemma 2.2 iv)) applies and proves that

$$
\inf _{\Sigma^{t} \cap \Sigma} w^{t} \geq \inf _{\partial\left(\Sigma^{t} \cap \Sigma\right)} w^{t} \geq 0
$$

which is non-negative because $\partial_{n} u \geq 0$ on the boundary of the cylinder and by construction $-1 \leq u \leq 1$. Then

$$
w^{t} \geq 0 \quad \text { on } \quad \Sigma^{t} \cap \Sigma \text { for } t \in\left(2 h-\delta_{0}, 2 h\right) .
$$

Let

$$
t^{*}=\inf \left\{t, \forall s \geq t, w^{s} \geq 0 \quad \text { on } \quad \Sigma^{s} \cap \Sigma\right\} .
$$

By definition

$$
w^{t^{*}} \geq 0 \quad \text { on } \quad \Sigma^{t^{*}} \cap \Sigma
$$

Step 2. Continuation. Let us assume that $t^{*}>0$. We want to prove that we can then decrease the parameter $t$. On the one hand the strong maximum principle implies that

$$
w^{t^{*}}>0 \text { on } \Sigma^{t} \cap \Sigma
$$

and then for every $\delta>0$, on the compact set

$$
K_{\delta}:=\left\{x \in \Sigma^{t^{*}} \cap \Sigma, d\left(x, \partial\left(\Sigma^{t} \cap \Sigma\right)\right) \geq \delta\right\},
$$

we have

$$
w^{t^{*}} \geq \delta^{\prime}>0 \quad \text { on } \quad K_{\delta}
$$

for some small enough $\delta^{\prime}$ and then

$$
w^{t^{*}-s} \geq \delta^{\prime} / 2>0 \quad \text { on } \quad K_{\delta} \quad \text { for } \quad s \in\left(0, s^{*}\right),
$$

for some small enough $s^{*}$. Now for $\delta$ small enough, the complementar $\mathcal{C}_{\delta}$ of $K_{\delta}$ defined by $\mathcal{C}_{\delta}:=\left(\Sigma^{t} \cap \Sigma\right) \backslash K_{\delta}$ has a measure sufficiently small to apply the maximum principle on it (Lemma 2.2 iv)). This gives

$$
\inf _{\mathcal{C}_{\delta}} w^{t^{*}-s} \geq \inf _{\partial \mathcal{C}_{\delta}} w^{t^{*}-s} \geq 0
$$

which is non-negative for the same reasons as in (4.9). Then

$$
w^{t^{*}-s} \geq 0 \quad \text { on } \quad \Sigma^{t^{*}-s} \cap \Sigma \quad \text { for } \quad s \in\left(0, s^{*}\right) .
$$

This contradicts the definition of $t^{*}$. 
Step 3. Conclusion. Then $t^{*}=0$ and

$$
u^{t} \geq u \quad \text { on } \quad \Sigma^{t} \cap \Sigma \quad \text { for } \quad t \in(0,2 h),
$$

i.e.

$$
\partial_{n} u \geq 0 \quad \text { on } \quad \Sigma \text {. }
$$

Coming back to the notation $u_{t, h}$ and $\Sigma_{h}$ this proves the lemma.

To prove Theorem 1.7, we need a last lemma:

Lemma 4.6. Let $F$ be a $C^{1,1}(\mathbb{R})$ function such that

$$
\left\{\begin{array}{l}
F \geq 0 \quad \text { on } \quad \mathbb{R} \\
F>0 \quad \text { on } \quad(-1,1) \text { and } F(-1)=F(1)=0 .
\end{array}\right.
$$

Then there exists a unique solution $u_{0}$ to

$$
u_{0}^{\prime \prime}=F^{\prime}\left(u_{0}\right) \quad \text { and } \quad u_{0}^{\prime}>0,
$$

with $u_{0}(-\infty)=-1, u_{0}(+\infty)=1, u_{0}(0)=0$.

Proof of Lemma 4.6. For $F \in C^{2}(\mathbb{R})$ this lemma is a consequence of Lemma 3.2 in [4]. The proof of Lemma 3.2 essentially uses the fact that

$$
u_{0}^{\prime 2}=2 F\left(u_{0}\right)
$$

It is easy to check that $F \in C^{1,1}(\mathbb{R})$ is sufficient to get the desired result.

Proof of Theorem 1.7. We assume the existence of a function $v\left(x^{\prime}\right)$ on $\mathbb{R}^{n-1}$ as defined in the theorem and we will build a function $u\left(x^{\prime}, x_{n}\right)$ on $\mathbb{R}^{n}$. The key idea is to connect on $\mathbb{R}^{n}$ the two particular solutions $u_{0}\left(x_{n}\right)$ and $v\left(x^{\prime}\right)$ by a continuous curve of solutions on $\mathbb{R}^{n}$. To this end we recall that we introduce the following functions for $t \in[0,1]$ :

$$
U_{t}\left(x^{\prime}, x_{n}\right):=u_{0}\left(t u_{0}^{-1}\left(v\left(x^{\prime}\right)\right)+(1-t) x_{n}\right),
$$

which satisfies in particular

$$
\left\{\begin{array}{l}
U_{0}\left(x^{\prime}, x_{n}\right)=u_{0}\left(x_{n}\right) \\
U_{1}\left(x^{\prime}, x_{n}\right)=v\left(x^{\prime}\right)
\end{array}\right.
$$

Moreover, the functions $U_{t}$ satisfy

$$
\partial_{n} U_{t} \geq 0
$$

and the same symmetries as $v$, namely (1.5). We will build monotonic solutions on cylinders whose values on the boundary of the cylinder are $U_{t}$ and then take the limit as the cylinders tend to the whole space $\mathbb{R}^{n}$. 
From Proposition 4.4 we know the existence of a global minimizer $u_{t}$ on $\Sigma=B_{R}^{\prime}(0) \times \mathbb{R}$ which satisfies

$$
\left\{\begin{array}{lll}
\Delta u_{t}=F^{\prime}\left(u_{t}\right) & \text { on } & \Sigma \\
u_{t}=U_{t} & \text { on } & \partial \Sigma \\
\partial_{n} u_{t} \geq 0 & \text { on } & \Sigma \\
\left|u_{t}\right| \leq 1 & \text { on } & \Sigma .
\end{array}\right.
$$

Moreover, by Proposition 4.3, we know that such a function is unique. From this uniqueness, we deduce that $u_{t}$ has the same symmetries as $U_{t}$, i.e.

$$
\begin{aligned}
& u_{t}\left(x_{1}, \ldots,-x_{i}, \ldots, x_{n-1}, x_{n}\right)=u_{t}\left(x_{1}, \ldots, x_{i}, \ldots, x_{n-1}, x_{n}\right) \\
& \text { for } i=1, \ldots, n-1 .
\end{aligned}
$$

Standard elliptic estimates imply the compactness of the set of functions $\left\{u_{t}\right\}_{t \in[0,1]}$ for the topology of convergence in $C^{2}$-norms on every compact sets of $\Sigma$.

For each $t \in[0,1]$ we define the real $h_{t}$ by

$$
u_{t}\left(0, \ldots, 0, h_{t}\right)=v(0)
$$

Here from the strict monotonicity of $u_{t}$ for each $t \in[0,1)$ we deduce the uniqueness of $h_{t}$. On the contrary $h_{t}$ for $t=1$ can be any real number because $u_{t}\left(x^{\prime}, x_{n}\right)=v\left(x^{\prime}\right)$ for $t=1$. We will later prove the following:

Lemma 4.7. The map $t \longmapsto \partial_{n} u_{t}\left(0, \ldots, 0, h_{t}\right)$ is continuous for $t \in[0,1]$.

In particular we have

$$
\begin{cases}\partial_{n} u_{t}\left(0, \ldots, 0, h_{t}\right)=u_{0}^{\prime}\left(u_{0}^{-1}(v(0))\right)=\sqrt{2 F(v(0))} & \text { for } t=0 \\ \partial_{n} u_{t}\left(0, \ldots, 0, h_{t}\right)=0 & \text { for } t=1\end{cases}
$$

Now for every $\gamma \in\left(0, u_{0}^{\prime}\left(u_{0}^{-1}(v(0))\right)\right)$, we get, by continuity, the existence of a $t_{\gamma} \in(0,1)$ such that

$$
\partial_{n} u_{t_{\gamma}}\left(0, \ldots, 0, h_{t_{\gamma}}\right)=\gamma, \quad \text { where } \quad u_{t_{\gamma}}\left(0, \ldots, 0, h_{t_{\gamma}}\right)=v(0) .
$$

We then redefine the solution with the origin in $\left(0, \ldots, 0, h_{t_{\gamma}}\right)$ by

$$
u_{R}^{\gamma}\left(x^{\prime}, x_{n}\right):=u_{t_{\gamma}}\left(x^{\prime}, x_{n}+h_{t_{\gamma}}\right) \quad \text { on } \quad B_{R}^{\prime}(0) \times \mathbb{R} .
$$

We can now take the limit $R \rightarrow+\infty$, and up to extraction of a convergent subsequence $\left\{R^{\prime}\right\}$ we get that

$$
u_{R^{\prime}}^{\gamma} \rightarrow u^{\gamma} \quad \text { uniformly locally on compact sets of } \mathbb{R}^{n},
$$

and the functions $u^{\gamma}$ are still global minimizers and satisfy

$$
\begin{cases}\Delta u^{\gamma}=F^{\prime}\left(u^{\gamma}\right) & \text { on } \quad \mathbb{R}^{n} \\ \partial_{n} u^{\gamma} \geq 0 & \text { on } \quad \mathbb{R}^{n} \\ \partial_{n} u^{\gamma}(0)=\gamma & \text { and } u^{\gamma}(0)=v(0) .\end{cases}
$$


The fact that $u^{\gamma}$ is not a planar solution comes from the fact that $u^{\gamma}$ inherits the symmetries of $v$

$$
\begin{aligned}
& u^{\gamma}\left(x_{1}, \ldots,-x_{i}, \ldots, x_{n-1}, x_{n}\right)=u^{\gamma}\left(x_{1}, \ldots, x_{i}, \ldots, x_{n-1}, x_{n}\right) \\
& \text { for } i=1, \ldots, n-1
\end{aligned}
$$

and then if $u^{\gamma}$ would be planar, it should be equal to some function

$$
u_{1}\left(x_{n}+h\right) \quad \text { where } h \text { is defined by } \quad u_{1}(h)=u^{\gamma}(0)=v(0) .
$$

Moreover, $u_{1}(+\infty)$ and $u_{1}(-\infty)$ should be constant minimizers and then $u_{1}(+\infty)=1, u_{1}(-\infty)=-1$ and $u_{1}\left(x_{n}+h\right) \equiv u_{0}\left(x_{n}+h^{\prime}\right)$. But this is impossible because $\partial_{n} u^{\gamma}(0)=\gamma<u_{0}^{\prime}\left(u_{0}^{-1}(v(0))\right)$. Finally the fact that

$$
\partial_{n} u^{\gamma}>0 \text { on } \mathbb{R}^{n}
$$

is a consequence of the strong maximum principle (Lemma 2.2 i)) and the fact that $\partial_{n} u^{\gamma}(0)=\gamma>0$. This ends the proof of Theorem 1.7, modulo Lemma 4.7.

Proof of Lemma 4.7. Using the uniqueness and the strict monotonicity of $u_{t}$, it is easy to see that the map $t \longmapsto \partial_{n} u_{t}\left(0, \ldots, 0, h_{t}\right)$ is continuous for $t \in$ $[0,1)$. We will now prove that this map is continuous up to $t=1$, i.e. that $\lim _{t \rightarrow 1} \partial_{n} u_{t}\left(0, \ldots, 0, h_{t}\right)=0$. If it is not the case then we can find a sequence $\left(t^{\prime}\right)$ of reals which converge to 1 and such that

$$
\partial_{n} u_{t^{\prime}}\left(0, \ldots, 0, h_{t^{\prime}}\right) \longrightarrow m \neq 0 .
$$

Let us define the sequence of functions

$$
\tilde{u}_{t^{\prime}}\left(x^{\prime}, x_{n}\right)=u_{t^{\prime}}\left(x^{\prime}, x_{n}+h_{t^{\prime}}\right)
$$

which are global minimizers on $\Sigma$. Then again using the compactness of these new sequence of functions $\tilde{u}_{t^{\prime}}$ in the topology of convergence in $C^{2}$-norms on every compact set of $\Sigma$, we deduce, up to extraction of a convergent subsequence, the existence of a limit $\tilde{u}_{1}$ which is a global minimizer on $\Sigma$ and satisfies:

$$
m=\partial_{n} \tilde{u}_{1}(0) \quad \text { and } \quad \tilde{u}_{1}(0)=v(0)
$$

and

$$
\left\{\begin{array}{lll}
\Delta \tilde{u}_{1}=F^{\prime}\left(\tilde{u}_{1}\right) & \text { on } & \Sigma \\
\tilde{u}_{1}=\tilde{U}_{1} & \text { on } & \partial \Sigma \\
\partial_{n} \tilde{u}_{1} \geq 0 & \text { on } & \Sigma \\
\left|\tilde{u}_{1}\right| \leq 1 & \text { on } & \Sigma
\end{array}\right.
$$

with

$$
\tilde{U}_{1}\left(x^{\prime}, x_{n}\right)=u_{0}\left(u_{0}^{-1}\left(v\left(x^{\prime}\right)\right)+l\right), \quad \text { where } \quad l=\lim _{t^{\prime} \rightarrow 1}\left(1-t^{\prime}\right) h_{t^{\prime}} \in \mathbb{R} \cup\{ \pm \infty\} .
$$

Here we use the definition $u_{0}\left(u_{0}^{-1}\left(v\left(x^{\prime}\right)\right)+\infty\right)=1$ and $u_{0}\left(u_{0}^{-1}\left(v\left(x^{\prime}\right)\right)-\infty\right)=-1$. 
We have now essentially two cases:

i) Case $l=0$. From Proposition 4.3, we deduce that $\tilde{u}_{1}\left(x^{\prime}, x_{n}\right)=v\left(x^{\prime}\right)$. This implies, in particular, that $\partial_{n} \tilde{u}_{1}(0)=0$, which is in contradiction with $m \neq 0$.

ii) Case $l \neq 0$. Let us assume that $l \in(0,+\infty)$ (the same reasoning applies to the cases $l<0$ and $l=+\infty)$. Let us define

$$
\underline{\tilde{u}}_{1}\left(x^{\prime}\right)=\lim _{x_{n} \rightarrow-\infty} \tilde{u}_{1}\left(x^{\prime}, x_{n}\right) .
$$

Then $\underline{\tilde{u}}_{1}$ is a global minimizer on $\Sigma$ and by Lemma 3.2, it is a minimizer on $B_{R}^{\prime}(0)$. Now let us define the open set

$$
\Omega_{-}=\left\{\underline{\tilde{u}}_{1}<v\right\} .
$$

Because $l>0$,

$$
v<\tilde{U}_{1}=\underline{\tilde{u}}_{1} \quad \text { on } \quad \partial B_{R}^{\prime}(0) .
$$

Therefore, $\Omega_{-} \subset \subset B_{R}^{\prime}(0)$. Then either $\Omega_{-} \neq \emptyset$ or else the reasoning of Step 1 in the proof of Lemma 3.1 applies and gives

$$
E_{\Omega_{-}}(v)=E_{\Omega_{-}}\left(\underline{\tilde{u}}_{1}\right) .
$$

We then see that the function

$$
v_{+}=\left\{\begin{array}{lll}
\tilde{u}_{1} & \text { on } & \Omega_{-} \\
v & \text { on } & B_{R}^{\prime}(0) \backslash \Omega_{-}
\end{array}\right.
$$

is a minimizer on $B_{R}^{\prime}(0)$ with the same boundary conditions as $v$. In particular, we deduce that $v_{+}$satisfies

$$
\left\{\begin{array}{lll}
\Delta v_{+}=F^{\prime}\left(v_{+}\right) & \text {on } & B_{R}^{\prime}(0) \\
v_{+} \leq \underline{\tilde{u}}_{1} & \text { on } & B_{R}^{\prime}(0) .
\end{array}\right.
$$

Then the strong maximum principle (Lemma $2.2 \mathrm{i}$ )) implies that either $v_{+}=\underline{\tilde{u}}_{1}$ (which is impossible because of (4.10)), or $v<\underline{\tilde{u}}_{1}$. Consequently we deduce that

$$
\tilde{u}_{1}(0) \geq \underline{\tilde{u}}_{1}(0)>v(0)
$$

which is a contradiction.

This ends the proof of the lemma.

Acknowledgements. We are very grateful to Luigi Ambrosio for his help in revising the manuscript and for pointing out that the proof of Theorem 1.7 was incomplete in an earlier draft. We have added Lemma 4.7 to take care of this.

The second author would like to thank NATO and the Massachusetts Institute of Technology. 


\section{References}

1. Alama, S., Bronsard, L., Gui, C.: Stationary layered solutions in $\mathbb{R}^{n}$ for an Allen-Cahn system with multiple well potential. Calc. Var. Partial. Differ. Equ. 5, 359-390 (1997)

2. Alberti, G., Ambrosio, L., Cabré, X.: On a long-standing conjecture of E. De Giorgi: symmetry in $3 d$ for general nonlinearities and a local minimality property. Acta Appl. Math. 65, 9-33 (2001)

3. Alt, H.W., Caffarelli, L.A., Friedman, A.: Variational problems with two phases and their free boundaries. Trans. Am. Math. Soc. 282, 431-461 (1984)

4. Ambrosio, L., Cabré, X.: Entire solutions of semilinear elliptic equations in $\mathbb{R}^{3}$ and a conjecture of De Giorgi. J. Am. Math. Soc. 13, 725-739 (2000)

5. Angenent, S.B.: Uniqueness of the solution of a semilinear boundary value problem. Math. Ann. 272, 129-138 (1985)

6. Athanasopoulos, I., Caffarelli, L.A., Salsa, S.: Caloric functions in Lipschitz domains and the regularity of solutions to phase transition problems. Ann. Math. 143, 413-434 (1996)

7. Athanasopoulos, I., Caffarelli, L.A., Salsa, S.: Regularity of the free boundary in parabolic phase-transition problems. Acta Math. 176, 245-282 (1996)

8. Athanasopoulos, I., Caffarelli, L.A., Salsa, S.: Phase Transition Problems of Parabolic Type: Flat Free Boundaries Are Smooth. Commun. Pure Appl. Math. 51, 77-112 (1998)

9. Barlow, M.T.: On the Liouville property for divergence form operators. Can. J. Math. 50, 487-496 (1998)

10. Barlow, M.T., Bass, R., Gui, C.: The Liouville Property and a Conjecture of De Giorgi. Commun. Pure Appl. Math. 53, 1007-1038 (2000)

11. Berestycki, H., Caffarelli, L., Nirenberg, L.: Symmetry for elliptic equations in a half space. In: Boundary value problems for partial differential equations and applications, Lions, J.-L., et al. (eds.). RMA Res. Notes Appl. Math., pp. 27-42. Paris: Masson 1993

12. Berestycki, H., Caffarelli, L., Nirenberg, L.: Inequalities for second order elliptic equations with applications to unbounded domains I. Duke Math. J. 81, 467-494 (1996)

13. Berestycki, H., Caffarelli, L., Nirenberg, L.: Monotonicity for elliptic equations in unbounded Lipschitz domains. Commun. Pure Appl. Math. 50, 1089-1111 (1997)

14. Berestycki, H., Caffarelli, L., Nirenberg, L.: Further qualitative properties for elliptic equations in unbounded domains. Ann. Sc. Norm. Super. Pisa, Cl. Sci., IV. Ser. 25, 69-94 (1997)

15. Berestycki, H., Nirenberg, L.: On the method of moving planes and the sliding method. Bol. Soc. Bras. Mat. 22, 1-37 (1991)

16. Berestycki, H., Nirenberg, L.: Travelling fronts in cylinders. Ann. Inst. Henri Poincaré, Anal. Non Linéaire 9, 497-572 (1992)

17. Berestycki, H., Hamel, F., Monneau, R.: One-dimensional symmetry of bounded entire solutions of some elliptic equations. Duke Math. J. 103, 375-396 (2000)

18. Bombieri, E., De Giorgi, E., Giusti, E.: Minimal cones and the Bernstein problem. Invent. Math. 7, 243-268 (1969)

19. Caffarelli, L.A.: A Harnack Inequality Approach to the Regularity of Free Boundaries. Commun. Pure Appl. Math. 39, 541-545 (1986)

20. Caffarelli, L.A.: A Harnack Inequality Approach to the Regularity of Free Boundaries. Part I: Lipschitz Free Boundaries are $C^{1, \alpha}$. Rev. Mat. Iberoam. 3, 139-162 (1987)

21. Caffarelli, L.A.: A Harnack Inequality Approach to the Regularity of Free Boundaries. Part II: Flat Free boundaries are Lipschitz. Commun. Pure Appl. Math. 42, 55-78 (1989)

22. Caffarelli, L.A.: A Harnack Inequality Approach to the Regularity of Free Boundaries. Part III: Existence Theory, Compactness, and Dependence on X. Ann. Sc. Norm. Super. Pisa, Cl. Sci., IV. Ser. 15, 583-602 (1988) 
23. Caffarelli, L.A., Cordoba, A.: Uniform Convergence of a Singular perturbation Problem. Commun. Pure Appl. Math. 48, 1-12 (1995)

24. Caffarelli, L.A., Cordoba, A.: Phase transition: Uniform regularity of the transition layers. Preprint

25. Caffarelli, L., Garofalo, N., Segala, F.: A gradient bound for entire solutions of quasilinear equations and its consequences. Commun. Pure Appl. Math. 47, 1457-1473 (1994)

26. Clément, P., Sweers, G.: Existence and multiplicity results for a semilinear elliptic eigenvalue problem. Ann. Sc. Norm. Super. Pisa Cl. Sci., IV. Ser. 14, 97-121 (1987)

27. Dacorogna, B.: Direct Methods in the Calculus of Variations. Applied Mathematical Sciences 78. Springer 1978

28. Dal Maso, G., Modica, L.: A general theory of variational functionals. In: Topics in Functional Analysis 1980-81. Quaderno Scuola Normale Superiore Pisa, 149-221 (1981)

29. De Giorgi, E.: Convergence problems for functionals and operators. In: Proc. Int. Meeting on Recent Methods in Nonlinear Analysis, Rome 1978, De Giorgi, E., et al. (eds.), 131-188. Bologna: Pitagora 1979

30. Evans, L.C.: Partial Differential Equations. Graduate Studies in Mathematics 19. Am. Math. Soc. 1998

31. Fleming, W.H.: On the oriented Plateau problem. Rend. Circ. Mat. Palermo 9, 69-89 (1962)

32. Farina, A.: Some Remarks on a Conjecture of De Giorgi. Calc. Var. Partial Differ. Equ. 8, 233-245 (1999)

33. Farina, A.: Symmetry for solutions of semilinear elliptic equations in $\mathbb{R}^{n}$ and related conjectures. Ric. Mat. 48, 129-154 (1999)

34. Farina, A.: One-dimensional symmetry for solutions of quasilinear equations in $\mathbb{R}^{2}$. Boll. Unione Mat. Ital. Sez. B, Art. Ric. Mat. (8) 6, 685-692 (2003)

35. Farina, A., Akopian, V.: Sur les solutions radiales de l'équation $-\Delta u=u\left(1-|u|^{2}\right)$ dans $\mathbb{R}^{n}(N \geq 3)$. C. R. Acad. Sci., Paris, Sér. I, Math. 325, 601-604 (1997)

36. Federer, H.: Real flat chains, cochains and variational problems. Indiana Univ. Math. J. 24, 351-407 (1974)

37. Ghoussoub, N., Gui, C.: On a conjecture of De Giorgi and some related problems. Math. Ann. 311, 481-491 (1998)

38. Ghoussoub, N., Gui, C.: On De Giorgi's conjecture in Dimensions 4 and 5. Ann. Math. 157, 313-334 (2003)

39. Giaquinta, M., Hildebrandt, S.: Calculus of Variations I. Grundlehren der Math. Wissensch. 310. Berlin, New York: Springer 1996

40. Giusti, E.: Minimal Surfaces and Functions of Bounded Variation. Basel-Boston: Birkhäuser 1984

41. Hutchinson, J.E., Tonegawa, Y.: Convergence of phase interfaces in the Van Der Waals-Cahn-Hilliard theory. Calc. Var. Partial Differ. Equ. 10, 49-84 (2000)

42. Kawohl, B.: Symmetry or not? Math. Intell. 20, 16-22 (1998)

43. Luckhaus, S., Modica, L.: The Gibbs-Thompson relation within the gradient theory of phase transitions. Arch. Ration. Mech. Anal. 107, 71-83 (1989)

44. Miranda, M.: Superfici minime illimitate. Ann. Sc. Norm. Super. Pisa, Cl. Sci., IV. Ser. 4, 313-322 (1977)

45. Miranda, M.: Maximum principle and minimal surfaces. Ann. Sc. Norm. Super. Pisa, Cl. Sci., IV. Ser. 25, 667-681 (1997)

46. Modica, L.: $\Gamma$-convergence to minimal surfaces problem and global solutions of $\Delta u=$ $2\left(u^{3}-u\right)$. In: Proc. Int. Meeting on Recent Methods in Nonlinear Analysis, Rome 1978, De Giorgi, E., et al. (eds.). Bologna: Pitagora 1979

47. Modica, L.: A gradient bound and a Liouville theorem for nonlinear Poisson equations. Commun. Pure Appl. Math. 38, 679-684 (1985)

48. Modica, L.: Gradient theory of phase transitions with boundary contact energy. Ann. Inst. Henri Poincaré 4, 487-512 (1987) 
49. Modica, L.: The Gradient Theory of Phase Transitions and the Minimal Interface Criterion. Arch. Ration. Mech. Anal. 98, 123-142 (1987)

50. Modica, L.: Monotonicity of the energy for entire solutions of semilinear elliptic equations. Partial differential equations and the calculus of variations, Vol. II., Progr. Nonlinear Differential Equations Appl. 2, 843-850. Boston: Birkhäuser 1989

51. Modica, L., Mortola, S.: Un esempio di $\Gamma^{-}$-convergenza. Boll. Unione Mat. Ital. Sez. B 14, 285-299 (1977)

52. Modica, L., Mortola, S.: Some entire solutions in the plane of nonlinear Poisson equations. Boll. Unione Mat. Ital. 5, 614-622 (1980)

53. Morgan, F.: Area minimizing surfaces, faces of Grassmannians, and calibrations. Am. Math. Mon. 95, 813-822 (1988)

54. Morgan, F.: Calibrations and new singularities in area-minimizing surfaces: a survey. In: Variational Methods, Berestycki, H., et al. (eds.). Progress in Nonlinear Differential Equations and Applications 4, 329-342. Boston: Birkhäuser 1990

55. Owen, N.C., Rubinstein, J., Sternberg, P.: Minimizers and gradient flows for singularly perturbed bi-stable potentials with a Dirichlet condition. Proc. R. Soc. Lond., Ser. A, Math. Phys. Eng. Sci. 429, 533-554 (1990)

56. Pacard, F., Ritoré, M.: From constant mean curvature hypersurfaces to the gradient theory of phase transitions. Preprint 2002

57. Simon, L.: Entire solutions of the minimal surface equation. J. Differ. Geom. 30, 643-688 (1989)

58. Simon, L.: The minimal surface equation. In: Geometry V: Minimal surfaces, Osserman, R., et al. (eds.). Transl. from the Russian. Encycl. Math. Sci. 90, 239-266. Berlin: Springer 1997 